\title{
Modular Spaces Topology
}

\author{
Ahmed Hajji \\ Laboratory of Mathematics, Computing and Application, Department of Mathematics, Faculty of Sciences, \\ Mohammed V-Agdal University, Rabat, Morocco \\ Email: hajid2@yahoo.fr
}

Received April 29, 2013; revised May 29, 2013; accepted June 7, 2013

Copyright (C) 2013 Ahmed Hajji. This is an open access article distributed under the Creative Commons Attribution License, which permits unrestricted use, distribution, and reproduction in any medium, provided the original work is properly cited.

\begin{abstract}
In this paper, we present and discuss the topology of modular spaces using the filter base and we then characterize closed subsets as well as its regularity.
\end{abstract}

Keywords: Topology of Modular Spaces; $\Delta_{2}$-Condition; Filter Base

\section{Introduction}

In the theory of the modular spaces $X_{\rho}$, the notion of $\Delta_{2}$-condition depends on the convergence of the sequences in modular space $X_{\rho}$. More precisely, it reads: for any sequence $\left(x_{n}\right)_{n \in \mathbb{N}}$ in $X_{\rho}$, if $\lim _{n \rightarrow+\infty} \rho\left(2 x_{n}\right)=0$, we have $\lim _{n \rightarrow+\infty} \rho\left(2 x_{n}\right)=0$. This condition has been used to study the topology of modular spaces, see J. Musielak [1], and to establish some fixed point theorems in modular spaces, see [2-7]. Some fixed point theorems without $\Delta_{2}$-condition can be found in $[8,9]$.

In this paper, we present a new equivalent form for the $\Delta_{2}$-condition in the modular spaces $X_{\rho}$ which is used to show that the corresponding topology is separate and to establish some associated topological properties, including the characterization of the $\rho$-closed subsets as well as its regularity. The present work is an improved English version of a pervious preprint in French [10].

\section{Preliminaries}

We begin by recalling some definitions.

Definition 2.1 Let $X$ be an arbitrary vector space over $K=\mathbb{R}$ or $\mathbb{C}$.

1) A functional $\rho: X \rightarrow[0,+\infty]$ is called modular if $\rho(x)=0$ implies $x=0$.

a) $\rho(-x)=\rho(x)$ for any $x \in X$ when $K=\mathbb{R}$, and

b) $\rho\left(\mathrm{e}^{i t} x\right)=\rho(x)$ for any real $t$ when $K=\mathbb{C}$.

c) $\rho(\alpha x+\beta y) \leq \rho(x)+\rho(y)$ for $\alpha, \beta \geq 0$ and $\alpha+\beta=1$.

2) If we replace c) by the following $\rho(\alpha x+\beta y) \leq \alpha \rho(x)+\beta \rho(y) \quad$ for $\quad \alpha, \beta \geq 0 \quad$ and $\alpha+\beta=1$, then the modular $\rho$ is called convex.

3) For given modular $\rho$ in $X$, the $X_{\rho}=\{x \in X / \rho(\lambda x) \rightarrow 0$ as $\lambda \rightarrow 0\}$ is called a modular space.

4) a) If $\rho$ is a modular in $X$, then

$$
|x|_{\rho}=\inf \left\{u>0, \rho\left(\frac{x}{u}\right) \leq u\right\}
$$

is a $F$-norm.

b) Let $\rho$ be a convex modular, then

$$
\|x\|_{\rho}=\inf \left\{u>0, \rho\left(\frac{x}{u}\right) \leq 1\right\}
$$

is called the Luxemburg norm.

\section{Topology $\tau$ in Modular Spaces}

In this section, we introduce the property $\tau_{0}$ for a modular $\rho$, which will be used to show that the corresponding topology, noted by $\mathcal{T}$, on modular space $X_{\rho}$ is separate, and to characterize their closed subsets.

We begin with the following

Proposition 3.1 Consider the family

$\mathcal{B}=\left\{B_{\rho}(0, r) / r>0\right\}$, where

$$
B_{\rho}(0, r)=\left\{x \in X_{\rho} / \rho(x)<r\right\} \text {. }
$$

Then

1) The family $\mathcal{B}$ is a filter base.

2) Any element of $\mathcal{B}$ is balanced and absorbing. Furthermore, if $\rho$ is convex, then any element of $\mathcal{B}$ is convex.

Proof.

1) $\mathcal{B}$ is a filter base. Indeed, we have 
a) $\varnothing \notin \mathcal{B}$ because any $B_{\rho}(0, r) \neq \varnothing$.

b) Let $B_{\rho}\left(0, r_{1}\right)$ and $B_{\rho}\left(0, r_{2}\right)$ be in $\mathcal{B}$ and set $r=\inf \left(r_{1}, r_{2}\right)$. Then, for any $z \in B_{\rho}(0, r)$ we have

$$
\left\{\begin{array}{l}
\rho(z)<r \leq r_{1} \\
\rho(z)<r \leq r_{2}
\end{array}\right.
$$

and therefore $z \in B_{\rho}\left(0, r_{1}\right) \cap B_{\rho}\left(0, r_{2}\right)$. That is

$$
B_{\rho}(0, r) \subset B_{\rho}\left(0, r_{1}\right) \cap B_{\rho}\left(0, r_{2}\right) .
$$

Hence $\mathcal{B}$ is a filter base for the existence of $B_{\rho}(0, r) \in \mathcal{B}$ such that

$$
B_{\rho}(0, r) \subset B_{\rho}\left(0, r_{1}\right) \cap B_{\rho}\left(0, r_{2}\right) .
$$

2) Let $B_{\rho}(0, r) \in \mathcal{B}$.

a) $B_{\rho}(0, r)$ is balanced. Indeed, for given $\alpha=\lambda \mathrm{e}^{\mathrm{i} \theta}$ with $\theta \in \mathbb{R}$ and $\lambda=|\alpha| \leq 1$, and given $x \in B_{\rho}(0, r)$, we have

$$
\rho(\alpha x)=\rho\left(\lambda \mathrm{e}^{i \theta} x\right)=\rho(\lambda x) \leq \rho(x)<r .
$$

This means that $\alpha x \in B_{\rho}(0, r)$.

b) $B_{\rho}(0, r)$ is absorbing. Indeed, for given $x \in X_{\rho}$ we have $\lim _{\lambda \rightarrow 0} \rho(\lambda x)=0$. Whence, for all $r>0$ there exists $\delta>0$, such that $0<\lambda<\delta$ and $\rho(\lambda x)<r$. Hence, there exists $\lambda>0$ such that $\lambda x \in B_{\rho}(0, r)$. This shows that $B_{\rho}(0, r)$ is absorbing.

Now, assume that $\rho$ is in addition convex and let $B_{\rho}(0, r) \in \mathcal{B}$. For given $x, y \in B_{\rho}(0, r)$ and $\lambda \in[0,1]$, we have

$$
\rho(\lambda x+(1-\lambda) y) \leq \lambda \rho(x)+(1-\lambda) \rho(y)<r,
$$

then

$$
\lambda x+(1-\lambda) y \in B_{\rho}(0, r) .
$$

Thence $B_{\rho}(0, r)$ is convex.

Definition 3.1 We say that $\rho$ satisfies the property $\tau_{0}$ if for all $\varepsilon>0$, there exist $L>0$ and $\delta>0$ such that $|\rho(y)-\rho(x)|<\varepsilon$ for every $x, y$ satisfying $\rho(x)<L$ and $\rho(x-y)<\delta$.

Theorem 3.1 Assume that the modular $\rho$ satisfies the property $\tau_{0}$. Then $X_{\rho}$ is a separate topological vector space.

Proof. In Proposition 3.1, we have seen that the family $\mathcal{B}$ is a filter base, and furthermore any element of $\mathcal{B}$ is balanced and absorbing. On the other hand, for any $B_{\rho}(0, r)$, there exists $\delta_{0}>0$ such that

$$
B_{\rho}\left(0, \delta_{0}\right)+B_{\rho}\left(0, \delta_{0}\right) \subset B_{\rho}(0, r) .
$$

In fact, let $\varepsilon ; r>\varepsilon>0$. Since $\rho$ satisfies the property $\tau_{0}$, there are $L>0$ and $\delta>0$, such that for $\rho(x)<L$ and $\rho(x-y)<\delta$ we have $|\rho(y)-\rho(x)|<\varepsilon$. Thus, if we set

$$
\delta_{0}=\inf (r-\varepsilon, L, \delta),
$$

we see that for $z=x+y \in B_{\rho}\left(0, \delta_{0}\right)+B_{\rho}\left(0, \delta_{0}\right)$ with

$$
\left\{\begin{array}{l}
\rho(x)<\delta_{0} \\
\rho(y)<\delta_{0} .
\end{array}\right.
$$

We obtain $y=z-x \in B_{\rho}\left(0, \delta_{0}\right)$. This implies $\rho(z-x)<\delta_{0} \leq \delta$ and $\rho(x)<\delta_{0} \leq L$. Thence

$$
\rho(z)<\varepsilon+\rho(x)<\varepsilon+\delta_{0} \leq \varepsilon+r-\varepsilon=r .
$$

This infers that $z \in B_{\rho}(0, r)$, and so

$$
B_{\rho}\left(0, \delta_{0}\right)+B_{\rho}\left(0, \delta_{0}\right) \subset B_{\rho}(0, r) .
$$

Hence the family $\mathcal{B}$ is a fundamental system of neighborhoods of zero, then the unique topology defined by $\mathcal{B}$ in $X_{\rho}$ is given by

$$
\begin{aligned}
\mathcal{T}= & \left\{G \neq \varnothing, G \subset X_{\rho} / \text { if } x \in G,\right. \\
& \text { then } \exists V \in \mathcal{B} \text { such that } x+V \subset G\} \cup\{\varnothing\},
\end{aligned}
$$

so that $X_{\rho}$ is a topological vector space.

To show that $\left(X_{\rho}, \mathcal{T}\right)$ is separate, let $x, y$ in $X_{\rho}$ such that $x \neq y$ and assume that for any $V_{x}$ neighborhood of $x$ and $V_{y}$ neighborhood of $y$ we have $V_{x} \cap V_{y} \neq \varnothing$. So that one can consider

$$
z \in\left(x+B_{\rho}\left(0, \frac{1}{n}\right)\right) \cap\left(y+B_{\rho}\left(0, \frac{1}{n}\right)\right)
$$

for certain $n \in \mathbb{N}^{*}$. Then

$$
\left\{\begin{array}{l}
\rho(x-z)<\frac{1}{n} \\
\rho(y-z)<\frac{1}{n} .
\end{array}\right.
$$

Since $\rho$ satisfies the property $\tau_{0}$, then there exist for any $\varepsilon>0$, two reals $L>0$ and $\delta>0$, such that $|\rho(y)-\rho(x)|<\frac{\varepsilon}{2}$ for every $x, y$ satisfying $\rho(x)<L$ and $\rho(y-x)<\delta$. Now, set $Y=y-x$ and $X=z-x$ and note that we have

$$
\left\{\begin{array}{l}
\rho(X)=\rho(x-z)<\frac{1}{n} \\
\rho(Y-X)=\rho(y-z)<\frac{1}{n} .
\end{array}\right.
$$

It follows that for any $n \in \mathbb{N}$ such that $\frac{1}{n} \leq \inf \left(L, \delta, \frac{\varepsilon}{2}\right)$, we have

$$
\rho(Y)=\rho(y-x)<\rho(z-x)+\frac{\varepsilon}{2} \leq \frac{\varepsilon}{2}+\frac{\varepsilon}{2}=\varepsilon .
$$

This infers that $\rho(y-x)<\varepsilon$, for arbitrary $\varepsilon>0$. Thus, $\rho(x-y)=0$ and then $x=y$, a contradiction since by hypothesis $x \neq y$. Therefore there exist neighborhoods $V_{x}$ of $x$ and neighborhood $V_{y}$ of $y$ such that 
$V_{x} \cap V_{y}=\varnothing$.

\section{$\tau$ Convergence and Characterization of $\tau$-Closed Subsets of $X_{\boldsymbol{\rho}}$}

We begin by recalling some needed definitions of the $\rho$-convergence and the $\rho$-closed subsets of the the modular space $X_{\rho}$ (see for examples [2-8]).

Definition 3.2 Let $X_{\rho}$ be a modular space.

1) A sequence $\left(x_{n}\right)_{n \in \mathbb{N}}$ in $X_{\rho}$ is said to be $\rho$-convergent to $x$, denoted by $x_{n} \stackrel{\rho}{\rightarrow} x$, if $\rho\left(x_{n}-x\right) \rightarrow 0$ as $n \rightarrow+\infty$.

2) A subset $B$ of $X_{\rho}$ is said to be $\rho$-closed if for any sequence $\left(x_{n}\right)_{n \in \mathbb{N}} \subset B$, such that $x_{n} \stackrel{\rho}{\rightarrow} x$, we have $x \in B$. We denote by $\bar{B}^{\rho}$ the closure of $B$ in the sense of $\rho$.

3) A modular $\rho$ is said to be satisfying the Fatou property, if $\rho(x-y) \leq \liminf \rho\left(x_{n}-y_{n}\right)$ as $x_{n} \stackrel{\rho}{\rightarrow} x$ and $y_{n} \stackrel{\rho}{\rightarrow} y$.

In this section, we define the $\tau$-convergence, the $\tau$ closed subsets of $X_{\rho}$, and we show that the topology defined by $\rho$-closed in the definition before, noted by $\tau_{1}$, and the topology $\tau$ are the same topology.

The naturel convergence in the sense of the topology $\tau$ and $\tau$-closed subsets of $X_{\rho}$ are given by the following definitions.

Definition 3.3 A sequence $\left(x_{n}\right)_{n \in \mathbb{N}}$ in $X_{\rho}$ is said to be convergent to $x$ in the sense of the topology $\tau$ (or simply $\tau$-convergent) if for any $\varepsilon>0$ there exists $N_{0} \in \mathbb{N}$ such that $x_{n} \in X+B(0, \varepsilon)$ whenever $n>N_{0}$.

Note that the property $\tau_{0}$ is a necessary condition to show the uniqueness of the limit when exists. Thus, the $\tau$-convergence need the property $\tau_{0}$ and it is easy to see that $\tau$-convergence and $\rho$-convergence are equivalent.

Definition 3.4 Let $\rho$ be a modular satisfying the property $\tau_{0}$. A subset $B$ of $X_{\rho}$ is said to be $\tau$-closed if and only if the complimentary of $B$ in $X_{\rho}$, noted by $C_{X_{\rho}}^{B}$, is an element of $\mathcal{T}$.

The following lemma shows that the property $\tau_{0}$ makes sense in the theory of modular spaces.

Lemma 3.1 Let $\rho$ be a modular and $X_{\rho}$ be $a$ modular space. Then $\rho$ satisfies the $\Delta_{2}$-condition if and only if $\rho$ satisfies the property $\tau_{0}$.

Proof. To prove "if", let $\left(x_{n}\right)_{n \in \mathbb{N}}$ be a sequence in $X_{\rho}$ such that $\rho\left(x_{n}\right) \rightarrow 0$ as $n \rightarrow+\infty$. This implies that for all $\varepsilon>0$, there exists $n_{0}$ such that for any $n>n_{0}$ we have

$$
\rho\left(x_{n}\right)<\inf \left(L, \delta, \frac{\varepsilon}{2}\right) .
$$

Now, take $X_{n}=x_{n}$ and $Y_{n}=2 x_{n}$, for any $n>n_{0}$. It follows

$$
\rho\left(X_{n}\right)=\rho\left(x_{n}\right)=\rho\left(Y_{n}-X_{n}\right)<\inf \left(L, \delta, \frac{\varepsilon}{2}\right) .
$$

This yields $\rho\left(Y_{n}\right)=\rho\left(2 x_{n}\right) \leq \frac{\varepsilon}{2}+\rho\left(x_{n}\right) \leq \varepsilon$ whenever $n>n_{0}$. Whence, the sequence $\left(\rho\left(2 x_{n}\right)\right)_{n \in \mathbb{N}}$ tends to zero as $n$ goes to $+\infty$, and therefore $\rho$ satisfies the $\Delta_{2}$-condition.

For "only if", let $\rho$ be a modular satisfying the $\Delta_{2}$-condition, and suppose that there exists $\alpha>0$ such that for any $L>0$ and for any $\delta>0$, there exist $x, y \in X_{\rho}$ satisfying $\rho(x)<L, \rho(x-y)<\delta$ and $|\rho(y)-\rho(x)| \geq \alpha$. In particular, for $L=\delta=\frac{1}{n}$ there exist $x_{n}, y_{n} \in X_{\rho}$ such that

$$
\begin{gathered}
\rho\left(x_{n}\right)<\frac{1}{n}, \rho\left(y_{n}-x_{n}\right)<\frac{1}{n} \text { and } \\
\left|\rho\left(y_{n}\right)-\rho\left(x_{n}\right)\right| \geq \alpha,
\end{gathered}
$$

which implies $\rho\left(x_{n}\right) \rightarrow 0$ and $\rho\left(y_{n}-x_{n}\right) \rightarrow 0$ as $n \rightarrow+\infty$. However, we have

$$
\begin{aligned}
\rho\left(y_{n}\right) & =\rho\left(\left(y_{n}-x_{n}\right)+x_{n}\right) \\
& \leq \rho\left(2\left(x_{n}-y_{n}\right)\right)+\rho\left(2 x_{n}\right) .
\end{aligned}
$$

Now, since $\rho$ satisfies the $\Delta_{2}$-condition, then $\rho\left(y_{n}\right) \rightarrow 0$ as $n \rightarrow+\infty$. It follows that

$$
\left|\rho\left(y_{n}\right)-\rho\left(x_{n}\right)\right| \rightarrow 0 \text { as } n \rightarrow+\infty,
$$

which contradicts the fact that $\left|\rho\left(y_{n}\right)-\rho\left(x_{n}\right)\right| \geq \alpha>0$ for any $n \in \mathbb{N}$. Finally, for all $\varepsilon>0$, there are $L>0$ and $\delta>0$ such that if $\rho(x)<\delta$ and $\rho(y-x)<\delta$, we have $|\rho(y)-\rho(x)|<\varepsilon$. This completes the proof of Lemma 3.1.

In the following theorem, we show that the $\tau$-topology and the $\tau_{1}$-topology are the same.

Theorem 3.2 Let $\rho$ be a modular satisfying the $\Delta_{2}$ condition and $F \subset X_{\rho}$, then $F$ is $\tau$-closed if and only if $F$ is $\rho$-closed.

The following result is needed to show Theorem 3.2.

Proposition 3.2 Let $\rho$ be a modular satisfying the $\Delta_{2}$-condition and $\mathrm{F}$ a $\tau$-closed subset of $X_{\rho}$. Then

$$
x \in F \Leftrightarrow \forall \varepsilon>0, B_{\rho}(x, \varepsilon) \cap F \neq \varnothing .
$$

Proof. For $x \in X_{\rho}$, we have

$x \notin F \Leftrightarrow x \in C_{X_{\rho}}^{F}, C_{X_{\rho}}^{F}$ is an open set of the $\tau$-topology

$$
\begin{aligned}
& \Leftrightarrow \exists B_{\rho}(0, \varepsilon) \in \mathcal{B} / x+B_{\rho}(0, \varepsilon)=B_{\rho}(x, \varepsilon) \subset C_{X_{\rho}}^{F} \\
& \Leftrightarrow \exists \varepsilon>0, \text { such that } B_{\rho}(x, \varepsilon) \cap F=\varnothing .
\end{aligned}
$$


Finally,

$$
x \in F \Leftrightarrow \forall \varepsilon>0, B_{\rho}(x, \varepsilon) \cap F \neq \varnothing .
$$

Proof of Theorem 3.2. Let $F$ be $\tau$-closed and $\left(x_{n}\right)_{n \in \mathbb{N}}$ be a sequence in $F$ such that $x_{n} \rightarrow x$. Then, for any $\varepsilon>0$, there exists $n_{0} \in \mathbb{N}$ such that for every $n>n_{0}$, we have $x_{n} \in B(x, \varepsilon)$. This implies that

$$
\forall \varepsilon>0, B(x, \varepsilon) \cap F \neq \varnothing .
$$

Whence, making use of Proposition 3.1, we get that $x \in F$.

Conversely, assume that $F$ is not $\tau$-closed, then $C_{X_{\rho}}^{F}$ is not an open set for the $\tau$-topology. There exists then $x \in C_{X_{\rho}}^{F}$ satisfying $B_{\rho}(x, \varepsilon) \not \subset C_{X_{\rho}}^{F}$ and so $B(x, \varepsilon) \cap F \neq \varnothing$ for any $\varepsilon>0$. Therefore, for $\varepsilon=\frac{1}{k}$ there exists $x_{k} \in B_{\rho}\left(x, \frac{1}{k}\right) \cap F$. Thence, the obtained sequence $\left(x_{n}\right)_{n \in \mathbb{N}} \subset F$ satisfies $x_{n} \stackrel{\rho}{\rightarrow} x$. This implies $x \in F$, which is in contradiction with the fact that $x \in C_{X_{\rho}}^{F}$. In conclusion, $F$ is $\tau$-closed.

Remark 3.1 Observe that

$\rho$ satisfies the $\Delta_{2}$-condition

$\Leftrightarrow \rho$ satisfies the property $\tau_{0}$.

As consequence, we see that under the assumption that $\rho$ satisfies the $\tau_{0}$ property, we have

$\tau_{1}$ topology $\Leftrightarrow \tau$ topology.

Then definitions of $\rho$-convergence and $\rho$-closed subsets of $X_{\rho}$ need the hypothesis that $\rho$ satisfies the $\Delta_{2}$-condition.

The following result shows that the modular space $X_{\rho}$ is a regular space.

Theorem 3.3 Let $\rho$ be a modular satisfying the $\Delta_{2}-$ condition, $A$ be a $\tau$-closed subset of $X_{\rho}$ and $x_{0} \notin A$. Then there exists an open neighborhood $V_{x_{0}}$ of $x_{0}$ such that $V_{x_{0}} \cap A=\varnothing$.

In order to show the theorem above, we need the following result.

Proposition 3.3 Let $\rho$ be a modular satisfying the $\Delta_{2}$-condition and $A \subset X_{\rho}$. Then

$$
\rho(x, A)=\inf \{\rho(x-y), y \in A\}=0
$$

if and only if $x \in \bar{A}^{\rho}$, where $\bar{A}^{\rho}$ is the closure of $A$ for the $\tau$-topology.

Proof. We have

$$
\rho(x, A)=\inf \{\rho(x-y), y \in A\}=0 .
$$

Then for any $\varepsilon=\frac{1}{n}$, there exists $y_{n} \in A$ such that $\rho\left(x-y_{n}\right)<\frac{1}{n}$ this implies that there exists a sequence $\left(y_{n}\right)_{n \in \mathbb{N}} \subset A$ such that $y_{n} \stackrel{\rho}{\rightarrow} x$. Whence $x \in \bar{A}^{\rho}$.

Inversely, let $x \in \bar{A}^{\rho}$, then by Theorem 3.2, there exists a sequence $\left(y_{n}\right)_{n \in \mathbb{N}} \subset A$ such that $y_{n} \stackrel{\rho}{\rightarrow} x$, therefore, for any $\varepsilon>0$ there exists $n_{0}$ such that

$$
\rho(x, A) \leq \rho\left(x-y_{n}\right)<\varepsilon ; \forall n>n_{0} .
$$

Hence

$$
\rho(x, A)=0 .
$$

Proof of the Theorem 3.3. By Proposition 3.3, $x_{0} \notin A$ if and only if $\rho\left(x_{0}, A\right)=r>0$. Next, since $\rho$ satisfies the $\Delta_{2}$-condition then by Lemma 3.1, for $\varepsilon=\frac{r}{3}>0$, there exist $L>0$, and $\delta>0$ such that if $\rho(x)<L$ and $\rho(y-x)<\delta$ we have $|\rho(y)-\rho(x)|<\varepsilon$. Moreover, there exists $m_{0} \in \mathbb{N}^{*}$ such that $\frac{r}{m}<\inf (L, \delta)$ whenever $m>m_{0}$. Now, let $m_{1} \geq \max \left(3, m_{0}\right)$ and we consider the open neighborhood of $x_{0}$

$$
V_{x_{0}}=x_{0}+B_{\rho}\left(0, \frac{r}{m_{1}}\right) \text {. }
$$

Suppose next that $V_{x_{0}} \cap A \neq \varnothing$ and let $y \in V_{x_{0}} \cap A$. Since $A$ is closed we make use of Proposition 3.1 to exhibit a sequence $\left(y_{n}\right)_{n \in \mathbb{N}} \subset A$ such that $y_{n} \stackrel{\rho}{\rightarrow} y$. So that one considers $X_{n}=y-y_{n}$ and $Y_{n}=x_{0}-y_{n}$. Since $y_{n} \in A$ and $x_{0} \notin A$, then $\rho\left(Y_{n}\right) \geq r$. On the other hand, note that

$$
\rho\left(X_{n}\right)=\rho\left(y-y_{n}\right)<\frac{r}{m_{1}}<\inf (L, \delta),
$$

whenever $n>n_{0}$ and

$$
\rho\left(X_{n}-Y_{n}\right)=\rho\left(x_{0}-y\right)<\frac{r}{m_{1}}<\inf (L, \delta) .
$$

Therefore

$$
r \leq \rho\left(Y_{n}\right)<\rho\left(y-y_{n}\right)+\varepsilon \leq \frac{r}{m_{1}}+\frac{r}{3} \leq \frac{2 r}{3}
$$

whenever $n>n_{0}$, a contradiction. Thus $V_{x_{0}} \cap A=\varnothing$.

Remark 3.2 If $\rho$ satisfies Fatou property, then

$$
\overline{B(0, r)}=\overline{B_{\rho}}(0, r)=\left\{x \in X_{\rho} / \rho(x) \leq r\right\}
$$

is a closed ball of the topology $\tau$. We note by $B_{f}(x, r)$ all closed ball centered at $x$ with the radius $r>0$ (see [7]).

Corollary 3.1 Under the same hypotheses of Theorem 
3.3, and if the modular $\rho$ satisfies Fatou property, then ${\overline{V_{x_{0}}}}^{\rho} \cap A=\varnothing$.

Proof. Making appeal of Theorem 3.3, there exists $V_{x_{0}}=x_{0}+B_{\rho}\left(0, \frac{r}{m_{1}}\right)$ such that $V_{x_{0}} \cap A=\varnothing$. Then, we have ${\overline{V_{x_{0}}}}^{\rho}=x_{0}+B_{f}\left(0, \frac{r}{m_{1}}\right)$. Indeed, let $y \in{\overline{V_{x_{0}}}}^{\rho}$ and note that from Proposition 3.1, there exists a sequence $\left(y_{n}\right)_{n \in \mathbb{N}} \subset B_{f}\left(0, \frac{r}{m_{1}}\right)$ such that

$$
x_{0}+y_{n} \stackrel{\rho}{\rightarrow} y,
$$

which implies that $y_{n} \stackrel{\rho}{\rightarrow} y-x_{0}$. Indeed, it is easy to see that $Y_{n}=y_{n}-\left(y-x_{0}\right) \stackrel{\rho}{\rightarrow} 0$ and since $\rho$ satisfies the $\Delta_{2}$-condition we have also $X_{n}=2\left(y_{n}-\left(y-x_{0}\right)\right) \stackrel{\rho}{\rightarrow} 0$. Thence, for $\varepsilon>0$, there are $L>0$ and $\delta>0$ such that

$$
\rho\left(X_{n}\right)<\inf \left(L, \delta, \frac{\varepsilon}{2}\right)
$$

and

$$
\rho\left(Y_{n}-X_{n}\right)=\rho\left(Y_{n}\right)<\inf \left(L, \delta, \frac{\varepsilon}{2}\right),
$$

whenever $n \geq n_{0}$, then

$$
\begin{aligned}
\rho\left(Y_{n}\right) & =\rho\left(y_{n}-\left(y-x_{0}\right)\right) \\
& <\inf \left(L, \delta, \frac{\varepsilon}{2}\right)+\frac{\varepsilon}{2} \leq \frac{\varepsilon}{2}+\frac{\varepsilon}{2}=\varepsilon,
\end{aligned}
$$

whenever $n \geq n_{0}$. Therefore

$$
y_{n} \stackrel{\rho}{\rightarrow} y-x_{0} \in \overline{B_{\rho}\left(0, \frac{r}{m_{1}}\right)^{\rho}}=B_{f}\left(0, \frac{r}{m_{1}}\right) .
$$

It follows

$$
y=x_{0}+\left(y-x_{0}\right) \in x_{0}+B_{f}\left(0, \frac{r}{m_{1}}\right),
$$

and hence

$$
{\overline{V_{x_{0}}}}^{\rho} \subset x_{0}+B_{f}\left(0, \frac{r}{m_{1}}\right) .
$$

Inversely, let

$$
x_{0}+y \in x_{0}+B_{f}\left(0, \frac{r}{m_{1}}\right) .
$$

By Proposition 3.1, there exists $\left(y_{n}\right)_{n \in \mathbb{N}} \subset B_{\rho}\left(0, \frac{r}{m_{1}}\right)$ such that $y_{n} \stackrel{\rho}{\rightarrow} y$. Moreover, the sequence $\left(x_{0}+y_{n}\right)_{n \in \mathbb{N}} \subset V_{x_{0}}$ satisfying $x_{0}+y_{n} \stackrel{\rho}{\rightarrow} x_{0}+y$. Hence

$$
x_{0}+y \in{\overline{\bar{x}_{0}}}^{\rho} .
$$

Finally, we take the same arguments as in the proof of Theorem 3.3, we have

$$
{\overline{V_{x_{0}}}}^{\rho} \cap A=\varnothing .
$$

\section{REFERENCES}

[1] J. Musielak, "Orlicz Spaces and Modular Spaces," Lecture Notes in Mathematics, Vol. 1034, 1983.

[2] A. Ait Taleb and E. Hanebaly, "A Fixed Point Theorem and Its Application to Integral Equations in Modular Function Spaces," Proceedings of the American Mathematical Society, Vol. 128, 2000, pp. 419-426. doi:10.1090/S0002-9939-99-05546-X

[3] A. Razani and R. Moradi, "Common Fixed Point Theorems of Integral Type in Modular Spaces," Bulletin of the Iranian Mathematical Society, Vol. 35, No. 2, 2009, pp. 11-24.

[4] A. Razani, E. Nabizadeh, M. B. Mohammadi and S. H. Pour, "Fixed Point of Nonlinear and Asymptotic Contractions in the Modular Space," Abstract and Applied Analysis, Vol. 2007, 2007, Article ID: 40575.

[5] A. P. Farajzadeh, M. B. Mohammadi and M. A. Noor, "Fixed Point Theorems in Modular Spaces," Mathematical Communications, Vol. 16, 2011, pp. 13-20.

[6] M. A. Khamsi, "Nonlinear Semigroups in Modular Function Spaces," Thèse d'état, Département de Mathématiques, Rabat, 1994.

[7] M. A. Khamsi, W. Kozlowski and S. M.-Reich, "Fixed Point Theory in Modular Function Spaces," Nonlinear Analysis, Theory, Methods and Applications, Vol. 14, No. 11, 1990, pp. 935-953.

[8] F. Lael and K. Nourouzi, "On the Fixed Points of Correspondences in Modular Spaces," ISRN Geometry, Vol. 2011, 2011, Article ID: 530254. doi:10.5402/2011/530254

[9] M. A. Khamsi, "Quasicontraction Mappings in Modular Spaces without $\Delta_{2}$-Condition," Fixed Point Theory and Applications, Vol. 2008, 2008, Article ID: 916187.

[10] A. Hajji, "Forme Equivalente à la Condition $\Delta_{2}$ et Certains Résultats de Séparations dans les Espaces Modulaires," 2005. http://arXiv.org/abs/math.FA/0509482 